\title{
Chimie, chimistes et rationalisation sous les auspices du ministre du Commerce et de l’Industrie Étienne Clémentel (1917-1919)
}

\author{
Michel LETTÉ *
}

\begin{abstract}
Résumé : L'expression « guerre chimique » associée à la première guerre mondiale ne l'est pas seulement du fait de l'usage des gaz de combat sur le champ de bataille, mais aussi du fait de l'importance des chimistes engagés dans la conduite de la guerre industrielle. Pour nombre d'entre eux mobilisés par le ministère du Commerce et de I'Industrie, le conflit est l'occasion de collaborer à la préparation économique de l'après-guerre. Entre 1917 et 1919 , ces chimistes partisans d'une gestion technocratique de la politique industrielle s'associent à Étienne Clémentel, l'un des principaux acteurs de l'économie en guerre. Ensemble, ils élaborent un programme de refondation de la production française. Le projet est porté par la conviction qu'il n'y a pas d'autre voie possible que celle dans laquelle s'est engagée l'Allemagne avant-guerre : rationaliser la production, faire collaborer les scientifiques, l'administration et les industriels. Cet article retrace l'action de ces chimistes promoteurs de la rationalisation. II rend compte de leurs actions à la direction du Comité consultatif des arts et manufactures, du congrès du Génie civil, de la Société de chimie industrielle, et enfin à la rédaction du Rapport général sur l'industrie française.
\end{abstract}

Mots-clés : première guerre mondiale ; chimie et industrie ; rationalisation ; Étienne Clémentel ; technocratie.

Summary : The association of " chemical warfare » and the First World War is not only due to the use of poison gas on the battlefield, but also to the importance of chemistry and chemists in conducting the industrial war. For many chemists involved, the conflict was an opportunity to collaborate in preparing the post-war economy. Between 1917 and 1919, these chemists advocated a technocratic management of the industrial policy. Together with Étienne Clémentel, the Minister of Trade and a key figure in the war economy, they prepared a blueprint for the reconstruction of the French production. Their project was based on the firm conviction that there was no alternative to the path taken by Germany before the war: the rationalisation of industry, the collective organisation as well as the collaboration between scientists, government and industry. This article traces the commitments of these chemists convinced to be part of the rationalisation process of the industrial economy. It focuses on their role in the Advisory Committee on the Arts and Manufactures, the Congress of Civil Engineering, the Society of Industrial Chemistry, and finally their influence on the editors of the general report on the French industry.

Keywords : First World War ; chemistry and industry ; rationalisation ; Étienne Clémentel ; technocracy.

* Michel Letté, Laboratoire « Histoire des technosciences en société » (HT2S - EA 3716), CNAM, Case 1LAB10, 2, rue Conté, 75003 Paris - E-mail : michel.lette@lecnam.net 


\section{Introduction}

De Jean-Antoine Chaptal à Albin Haller, les chimistes n'ont cessé d'alerter l'administration et le gouvernement sur le rôle primordial de la science et de l'industrie chimique dans l'édification de la puissance industrielle du pays ${ }^{1}$. Dans l'intervalle, d'innombrables rapports exhortent la nation à retrouver le rang honorable auquel le génie français des fondateurs de la chimie moderne l'avait jadis élevée ${ }^{2}$. Peine perdue, ou presque. Le $8_{e}$ congrès international de Chimie appliquée ravive une fois de plus, en 1912, le sentiment d'une chimie française bien en peine de soutenir l'arrogance des succès commerciaux allemands ${ }^{3}$. Bien que ce secteur de l'économie ait été dynamique avant-guerre, ses contemporains persistent à le juger comme étant en retard face à son rival d'outre-Rhin ${ }^{4}$. Au seuil du conflit, la chimie est en quelque sorte devenue un étalon de l'écart de performance entre deux systèmes de collaboration entre l'État, la science et l'industrie ${ }^{5}$. II ne se dément pas sur les champs de bataille, rappelant l'importance stratégique de la chimie et de son industrie, tout comme l'impératif de repenser sa place dans l'économie.

Avant que la première guerre mondiale ne vienne toutefois mettre à l'épreuve les puissances européennes, la perception d'un décrochage ne se cantonne pas au seul secteur de la chimie mais concerne toute la production scientifique et l'organisation industrielle. La chimie ne constitue-t-elle pas, malgré tout, à ce moment précis, le champ d'activité et de savoirs où l'action de l'administration et des entrepreneurs s'impose avec le plus d'urgence ? Au travers de cet épisode bref mais intense d'une chimie mise sous tension, la conviction d'une nécessaire réorganisation s'est imposée, non seulement au sein des états-majors des armées, mais tout autant des chancelleries de l'économie industrielle ${ }^{6}$.

Au demeurant, l'intervention immédiate de l'État contribue à restructurer l'industrie chimique. Son action conduit même, au sortir du conflit, à une situation de suréquipement pour quelques secteurs clés de la production, tandis que quelques entreprises prospèrent ${ }^{7}$. Passé les premiers temps de l'urgence, l'administration s'attache toutefois à définir l'avenir, à déterminer les moyens de poursuivre la lutte sur le terrain économique ${ }^{8}$. Avec la remobilisation industrielle fin 1916, et surtout l'année suivante, une véritable fièvre technocratique s'empare des chimistes et de l'administration ${ }^{9}$. Fonctionnaires et savants s'engagent pour la reconstruction de l'économie, invoquant sur le mode incantatoire les principes de l'organisation rationnelle ${ }^{10}$. Car si la rationalisation était avant-guerre un champ de doctrines en formation, le conflit conforte les désirs de réalisation parmi les ingénieurs, les entreprises et les administrateurs de l'économie industrielle ${ }^{11}$.

Parmi les instigateurs de ce tournant de la rationalisation se trouvent nombre de chimistes de premier plan, devenus les fervents partisans d'une gestion technocratique de l'économie et de l'organisation sociale. Par leur implication auprès de l'administration du commerce et de l'industrie, ils inaugurent des pratiques nouvelles de collaboration avec l'État, assignent à la chimie d'autres fonctions économiques, et enfin confortent tant la légitimité de leur rôle que leur statut politique et social ${ }^{12}$.

Quels sont ces chimistes impliqués auprès du ministre Étienne Clémentel et leurs idéologies sous-jacentes ? Cet article retrace le réseau formé par ces chimistes réformateurs mais aussi les lieux et moments où s'élabore la promesse d'une refonte concomitante de la chimie et de l'économie industrielle. Le Comité consultatif des arts et manufactures s'avère être le foyer d'action de ces chimistes collaborateurs du ministre ${ }^{13}$. Constituant la direction de l'équipe des rédacteurs du rapport dit Clémentel, celle des animateurs du congrès du Génie civil et des fondateurs de la Société de chimie industrielle, ils incarnent les piliers sur lesquels s'érige la volonté d'une chimie intégrée à l'économie rationnelle. Le réseau des alliances que ces chimistes forment entre 1917 et 1919 agit comme l'une de ces arrière-cours parlementaires, qui exerce une influence sur le devenir de l'économie sociale et industrielle ${ }^{14}$. Selon ses 
promoteurs, elle doit s'ériger sur des bases technocratiques, confortant la présence des savants et des ingénieurs aux côtés des politiques ${ }^{15}$. Distincte d'une analyse de la guerre chimique proprement dite et de ses retombées en matière de restructuration de ce secteur de la production, cette contribution s'attache donc, partant de l'échelle restreinte d'un vaste projet tant politique qu'économique, à la part prise par les chimistes au sein de l'administration pour reconstruire l'après-guerre industriel.

\section{Une guerre chimique?}

La première guerre mondiale a été, à plusieurs titres, qualifiée de guerre chimique ${ }^{16}$. Au jeu de l'histoire contrefactuelle, on peut gravement sourciller et rappeler que l'issue du conflit aurait pris une tout autre tournure si la chimie n'avait pu répondre aux urgences d'août et septembre 1914. De même si les chimistes n'avaient pu déployer les trésors d'ingéniosité dont ils ont fait preuve pour compenser les défaillances. En ce sens, la guerre fut chimique surtout parce que la chimie répondait aux besoins de production d'à peu près toutes les matières nécessaires à l'équipement des armées comme des populations. Extraction minière, traitement des substances, élaboration de matières de substitution, analyse et synthèse des composés, contrôle de la qualité, toutes ces opérations forment l'étendue sans limites du périmètre de la chimie appliquée, des arts et de l'industrie. La chimie est partout cruciale. Elle est de tous les secteurs d'activité, conditionne en grande partie l'amélioration des procédés. Pour ces raisons, l'hégémonie économique et commerciale de la chimie au XIXe siècle la désignait comme la reine des sciences utiles, et les chimistes comme les contributeurs majeurs à l'innovation industrielle ${ }^{17}$.

Pour autant, la guerre n'a pas plus été chimique qu'elle n'a été mécanique, scientifique, industrielle ou même psychologique, et chacun de ces ordres de considération peut se disputer (ou plutôt leurs historiens) une part majeure de la victoire, sans pour autant assigner à l'un de ces facteurs le statut de déterminant décisif. Disons-le d'emblée, la chimie en tant que telle ne fait ni plus ni moins l'objet d'une focalisation de la part des élites techniques et administratives, en comparaison, par exemple, de la construction mécanique ou de la métallurgie. L'ingénieur et industriel Auguste Rateau peut ainsi affirmer en 1918 : " Nous devons nous préparer à soutenir dans un futur plus ou moins lointain un nouveau choc et ne pas perdre de vue que si par malheur il survient, l'industrie mécanique sera sans doute avec l'industrie chimique un facteur de la lutte encore plus décisif qu'aujourd'hui ${ }^{18}$. " Ni l'une ni l'autre de ces sciences ou industries ne peuvent être en effet distinguées au sein d'une économie intégrée. Ce qui ne veut nullement dire que des évolutions significatives n'ont pas eu lieu dans leurs domaines respectifs. Le bilan, par exemple, en matière de réorganisation de la recherche en chimie ou de son enseignement après la guerre n'est certes pas négligeable, mais la révolution escomptée par certains n'a pas vraiment eu lieu ${ }^{19}$.

En revanche, la démonstration d'une faiblesse manifeste de la chimie française a été un puissant levier de mobilisation. Plus encore avec l'usage inauguré en 1915 des gaz de combat 20. La chimie s'est parée un temps des vertus mortifères de l'arme définitive, même si elle s'est avérée être surtout un instrument de terreur et de propagande massive ${ }^{21}$. Le caractère inéluctable de la chimie a cependant suffisamment imprégné les esprits pour faire de son industrie un étendard de la reconstruction économique et commerciale d'après-guerre face à l'Allemagne ${ }^{22}$. L'expression " guerre chimique » ne se réduit donc pas à l'utilisation des gaz sur les champs de bataille ou au rôle d'une science décisive entre toutes, mais renvoie à une mobilisation des chimistes qui ne se dément pas une fois la paix conclue ${ }^{23}$. Investis de la mission de reconstruire l'un des secteurs vitaux de l'économie, les chimistes revendiquent un peu plus qu'à l'accoutumée une participation directe aux destinées de l'industrie, et par exemple de se voir confier la direction de ses principales entreprises ${ }^{24}$. 


\section{Une chimie sur le champ de bataille}

Quel que soit le camp considéré, la chimie s'est révélée primordiale pour la conduite de la guerre ${ }^{25}$. En France, comme au sein des autres nations belligérantes, l'administration civile et militaire impose autant qu'elle le peut aux agents économiques la coordination de la production et des importations pour satisfaire des besoins toujours plus importants, tant en variété qu'en quantité ${ }^{26}$. Dès le début des hostilités, les services de l'État mobilisent de part et d'autre laboratoires et usines chimiques, pourvoient au remplacement rapide de matières premières vite indisponibles ${ }^{27}$. La démarche exige de battre le rappel des compétences détenues par des chimistes en nombre jugé déjà insuffisant avant-guerre ${ }^{28}$. La loi Dalbiez de 1915 permet ainsi de rappeler à l'arrière ceux dont l'industrie a cruellement besoin ${ }^{29}$.

Avec le besoin vital de munitions et d'explosifs, la chimie figure parmi les premières industries mobilisées. Le dirigisme impose par exemple, en 1915, à l'industrie gazière, d'extraire le benzène et le toluène de ses fabrications afin de disposer de matière pour la production de ses dérivés. Auparavant, les houilles n'étaient pas systématiquement distillées, ni les gaz débenzolés. Les benzols disponibles étaient vendus à l'Allemagne, à laquelle on achetait en retour les produits semi-finis nécessaires à la fabrication de matières colorantes. La guerre met fin à cette situation de dépendance dans laquelle ces options initiales l'avaient maintenue. Une commission de carbonisation instituée au ministère du Commerce et de l'Industrie est même chargée de rechercher les moyens d'accroître les quantités de houilles distillées, et par conséquent celles des sous-produits de la distillation.

Mais l'action de l'administration porte d'abord sur l'organisation même de la production et des importations à partir d'une instance centrale de coordination. L'Office des produits chimiques et pharmaceutiques est la première du genre, créée pour la durée de la guerre par décret, le 17 octobre 1914, au sein du ministère du Commerce et de l'Industrie ${ }^{30}$. II a pour mission de constater les quantités disponibles de produits chimiques et pharmaceutiques, d'évaluer leur production et d'assurer les approvisionnements et leur répartition. II a également pour fonction de contribuer au développement d'une production plus intense et d'encourager la fabrication des produits qui n'existaient pas avant la guerre, notamment les substances synthétiques. Leur fabrication est par ailleurs étroitement surveillée par le ministère de l'Armement.

L'impulsion préfigure la politique des consortiums mise en place peu avant la fin de la guerre dans le cadre des accords interalliés pour la fourniture des matières et équipements, mais aussi dans la perspective d'instaurer des relations plus organiques entre l'État, son administration, les industriels et le patronat après les hostilités. Les groupements d'entreprises d'une même production existaient certes bien avant la guerre, tel le Syndicat général des produits chimiques regroupant des associations patronales ${ }^{31}$, mais il faut attendre la création de l'Union des industries chimiques, en 1921, résultat direct de l'expérience de collaboration avec l'administration durant la guerre, pour les voir tenir un rôle significatif dans la conduite d'une politique économique en lien avec l'État ${ }^{32}$.

Dans les premiers temps de l'action, les initiatives se concrétisent donc sur le terrain de la coordination des potentiels de production et des besoins. Parmi les réalisations industrielles suscitées par l'administration, l'entrepreneur Donat Agache construit, en 1915, une usine d'acide sulfurique à Port-de-Bouc afin d'alimenter la poudrerie nationale de Saint-Chamas. À la demande du ministère du Commerce et de celui de la Guerre, les établissements Poulenc Frères se lancent dans la production synthétique de phénol et d'acide phénique. De même, la Compagnie nationale des matières colorantes est fondée le 31 janvier $1917^{33}$. L'entreprise construit deux usines, l'une à Villers-Saint-Paul, qui ne fut opérationnelle qu'en 1919, l'autre à Oissel. La société Minerais et Métaux est constituée en 1917 sur impulsion du ministère du Commerce et de l'Industrie, avec les gouvernements anglais et belge. Elle regroupe plusieurs dizaines de compagnies françaises d'exploitation. D'autres opérations d'envergure, d'autres 
regroupements, rachats et restructurations sont menés sous l'égide du gouvernement, notamment dans le domaine de la fabrication des produits azotés, pour se traduire par la création, en 1924, de l'Office national industriel de l'azote ${ }^{34}$.

C'est cependant à un programme de plus grande ampleur que s'attèlent les chimistes avec l'administration de guerre, visant à terme la constitution d'un véritable ministère de l'Économie nationale dont la vocation ne se limiterait pas à organiser le seul secteur de la chimie mais bien l'ensemble de l'industrie.

\section{Le culte de la rationalisation en partage}

Après une phase pragmatique d'implication des services techniques de l'État pour répondre aux urgences du moment, les services de guerre s'organisent afin de poursuivre un conflit qui, à l'évidence, s'est installé pour durer ${ }^{35}$. Parmi ses acteurs majeurs, le ministère du Commerce devient l'un des centres bureaucratiques de la guerre économique 36. II exerçait auparavant à l'ombre des Affaires étrangères et se contentait de gérer les intérêts nationaux au travers des importations et des exportations. Avec la première guerre mondiale, il s'impose comme l'un des dispositifs clés d'une organisation étatique où s'expérimente l'intervention directe. Un ambitieux programme de reconstruction s'élabore entre 1917 et 1919, donnant lieu à des réformes législatives et administratives, et à des réalisations techniques ${ }^{37}$. Toutes ne seront pas pérennes, mais toutes auront profondément transformé le rôle comme la fonction de ce ministère dans l'après-guerre, et, avec lui, la structure même de l'administration ${ }^{38}$. Ses actions ont contribué à orienter durablement la politique industrielle et commerciale de la France pour l'engager dans la voie de l'économie administrée ${ }^{39}$.

Étienne Clémentel arrive au ministère du Commerce le 29 octobre $1915^{40}$. Confronté à la désorganisation de la production, il engage la refonte des plans d'approvisionnement en armes et de réquisition, adopte un mode de gestion plus autoritaire des relations entre le gouvernement et les agents économiques du pays. Avec Albert Thomas et Louis Loucheur, il domine l'administration d'une économie de guerre, tout en délimitant les contours de la reconstruction ${ }^{41}$. Car telle est leur ambition : doter le pays après-guerre des cadres administratifs permettant de conduire le programme d'une réorganisation totale de l'industrie, dont un ministère de l'Économie et de la Production nationale serait l'inspirateur et les industriels les exécutants.

Comme ses contemporains, Clémentel est animé par la certitude qu'à la différence de la France, l'Allemagne s'était préparée à une guerre industrielle, et qu'elle compte bien la poursuivre une fois la paix revenue ${ }^{42}$. Cette supériorité des armées du Reich et la menace d'une expansion industrielle et commerciale de l'Allemagne sont largement attribuées tant aux capacités d'organisation de la nation allemande qu'à l'indiscipline des Français qui seraient trop épris de liberté ${ }^{43}$. C'est donc à l'édification d'une économie rationnelle que Clémentel voue son action, inspirée par l'organisation allemande comme par la fabrication en série aux États-Unis, une économie guidée par les savoirs experts produits par les savants et pratiquée par les ingénieurs ${ }^{44}$. Cette économie rationnelle qu'il appelle de ses vœux suppose cependant une évolution des mœurs politiques confinant à la technocratie ${ }^{45}$. Assumant son rôle d'administrateur d'une économie en guerre, Clémentel se fait planificateur et partisan de l'organisation collective, de l'économie administrée, de la doctrine et du culte de la rationalisation, artisan d'une refonte des rouages de l'économie par l'institutionnalisation des liens instaurés entre administration publique, acteurs et agents de l'économie ${ }^{46}$.

À l'occasion du remaniement de ses services techniques qu'il présente en septembre 1917, Clémentel rappelle la vocation de son administration : préparer le programme d'action économique et veiller à son application ${ }^{47}$. Parmi ses points inédits figure la proposition d'une extension considérable des services de l'enseignement technique, de manière à obtenir le 
rendement le plus élevé possible de l'industrie et de la main-d'œuvre nationale, mais aussi la création de laboratoires d'État qui assureraient la liaison entre la science et l'industrie et contribueraient à développer dans les usines et manufactures l'emploi des procédés techniques les plus perfectionnés ${ }^{48}$. C'est au sein même de la direction technique de son ministère que deux chimistes parmi les plus influents du moment prennent place afin de guider les travaux: Henry Le Chatelier et Albin Haller ${ }^{49}$.

Tous deux incarnent des écoles et des traditions, des conceptions de la chimie et des visions politiques qui s'opposent sur bien des aspects, mais pour l'heure, ils s'unissent dans un élan commun visant l'alliance de la chimie et de la rationalisation d'une économie en guerre ${ }^{50}$.

Le conseil technique est l'instance supérieure de cette organisation nouvelle. Sa direction est confiée à Léon Guillet, disciple de Le Chatelier ${ }^{51}$. Le conseil se réunit pour la première fois le 10 décembre 1917. Clémentel le considère comme le conseil d'administration de l'entreprise de reconstitution de l'économie française, son rôle étant de l'éclairer avant qu'il ne prenne ses décisions sur les questions générales qui lui sont soumises ${ }^{52}$.

\section{Les chimistes aux commandes}

La préparation économique et industrielle de l'après-guerre exige un travail considérable de production et d'agrégation de données, la mobilisation de spécialistes capables d'assurer leur exploitation, de conduire les études et les analyses, de rédiger les avis autorisés. Disposant de peu de moyens mais de plus en plus de responsabilités, Clémentel fédère les initiatives éparses et particulières, s'assure de la collaboration active des organisations auxiliaires, des experts qualifiés surtout, afin de disposer des informations indispensables à la prise de décision dans les domaines concernés, c'est-à-dire la plupart des registres de l'économie nationale. II démarche les associations savantes et professionnelles, les sociétés académiques comme les groupements d'ingénieurs et d'entreprises, telle la Société d'encouragement pour l'industrie nationale, dont Clémentel préside la séance du 5 mai 1917.

C'est au Comité consultatif des arts et manufactures, principal organe de production de l'expertise, qu'il revient d'élaborer le plan de reconstitution de l'économie industrielle. Ce comité était, depuis 1880, composé de seize membres pris dans le Conseil d'État, l'Académie des sciences, les corps des Ponts-et-Chaussées et des Mines, le commerce et l'industrie. Clémentel lui attribue, le 4 avril 1917, des fonctions et des prérogatives qu'il n'avait jamais eues. Sa composition est remaniée afin d'y intégrer notamment les chimistes ; il passe de seize à vingt-quatre membres permanents, auxquels sont associés des titulaires pour la durée de la guerre, puis des enquêteurs reconnus pour leurs hautes compétences. C'est au total plus d'une centaine d'experts, d'ingénieurs et de savants qui forme l'équipe des rapporteurs coordonnés par Guillet et le Comité consultatif des arts et manufactures ${ }^{53}$. Ce dernier est désormais structuré selon quatre sections principales (métallurgie et construction métallurgique ; textiles ; industries chimiques et connexes ; industries diverses), elles-mêmes divisées en sections secondaires, dédiées à une industrie, un secteur d'activité ou une matière première ${ }^{54}$. Haller préside le Comité. II est alors membre de l'Académie des sciences, professeur à la Sorbonne et directeur de l'École de physique et de chimie industrielles. Deux vice-présidents lui sont associés, dont Le Chatelier, également membre de l'Académie des sciences et professeur à la Sorbonne. Les sections sont respectivement placées sous la responsabilité de Le Chatelier, de Gabriel Chandèze, du chimiste Théophile Schloesing et d'Arsène d'Arsonval. La franche prédominance de la chimie et des chimistes dans la direction de ce comité d'experts n'échappera à personne. Sur un total de 87 études menées durant la période, 52 sont ainsi dédiées explicitement à la chimie et à ses domaines connexes ${ }^{55}$. Le constat n'a rien de surprenant. L'économie, l'industrie et la guerre sont assurément " chimiques ". La chimie n'est pas tant ici pensée pour elle-même que pour son rôle de socle commun à l'ensemble de la production rationnelle. Dans cette perspective de reconfiguration 
de l'économie, la logique du discours comme de l'action reste celle d'un vaste programme de redéfinition des bases technocratiques sur lesquelles doit s'ériger la reconstruction de l'économie.

C'est d'ailleurs en ces termes que Clémentel s'adresse à Le Chatelier et Haller lors de la séance d'ouverture des travaux du Comité, le 19 mai 1917 : "C'est à une oeuvre d'action que je vous convie. Vous avez à construire un instrument de travail propre à donner à la France une situation économique qui assurera sa force et sa grandeur dans l'avenir, par la puissance de ses moyens de productivité, par son activité commerciale et par l'habilité de ses méthodes. "Leur but, précise-t-il, " est de centraliser, examiner, contrôler et coordonner toutes les données relatives à la production nationale et de déterminer les mesures à prendre et les initiatives à provoquer en vue d'en améliorer les conditions après la conclusion de la paix " ${ }^{56}$. Le principe reste désormais de mobiliser toutes les compétences expertes disponibles et, pour cela, d' " utiliser une part des forces vives du pays au développement rationnel de notre industrie 57 ». Nul doute que les ressources invoquées sont à rechercher auprès des industriels, des scientifiques et des ingénieurs. Parmi les associations enclines à se faire technocrates, la Société des ingénieurs civils est celle sur laquelle Clémentel compte le plus. C'est à elle qu'il confie la mission de produire collectivement les études sur les moyens d'édifier une économie rationnelle.

\section{Déléguer l'expertise au congrès du Génie civil}

La Société des ingénieurs civils, fondée en 1848, rassemble, comme son nom l'indique, les ingénieurs civils, mais auxquels s'adjoignent, à partir de 1885, les ingénieurs de l'État. Sous l'impulsion notamment de Le Chatelier, Clémentel préside, le 3 novembre 1916, la séance de cette honorable société au cours de laquelle il sollicite la collaboration de ses membres. II leur demande de contribuer à la préparation de l'économie d'après-guerre, considérant que " l'avenir [...] impose une collaboration étroite entre la science et l'industrie : la science doit descendre de sa tour d'ivoire [...] elle doit pénétrer l'industrie, s'unir à elle intimement et [comprendre] que cette union est la base des conquêtes futures ". Ce langage un brin charnel dit toute la force de son désir d'une pleine collaboration des ingénieurs, représentants suprêmes de l'alliance de la science et de l'industrie, à la base même de l'économie rationnelle.

À sa demande, la Société des ingénieurs civils prend, en février 1917, l'initiative de convoquer un congrès national, c'est-à-dire l'organisation par la revue Le Génie civil de conférences sur la préparation de l'après-guerre. $Y$ sont conviés non seulement les membres de la Société, mais également tout ce que la communauté nationale compte de compétences techniques sur les questions d'économie industrielle ${ }^{58}$. Le principe est celui de la délibération sur la base de rapports dûment renseignés. Dans ce contexte de liens étroits avec le Comité consultatif des arts et manufactures, sa mission est de susciter, de réaliser, de rassembler et de discuter toutes les études techniques utiles à la préparation de la " contre-offensive d'après-guerre ». Ses organisateurs se fixent l'objectif de "réaliser entre les industriels et les savants une collaboration de plus en plus étroite, collaboration qui a donné une si grande force à l'industrie allemande et qui est une idée française 59 ». Leurs travaux forment l'expertise utile à la rédaction d'un rapport général sur l'industrie.

Le congrès se tient à Paris du 18 au 23 mars 1918 ; y contribuent plus de 1500 ingénieurs et experts. L'exposé des travaux se divise selon trois sections principales, faisant la part belle aux questions de rationalisation de l'économie : organisation rationnelle du travail industriel ; hygiène et prévoyance sociale ; législation industrielle. Lors de son discours d'inauguration, Clémentel accorde aux ingénieurs le " pouvoir de centraliser tous les travaux destinés à la préparation du programme économique de la France nouvelle », dont le cadre général est fixé par « la simplification des méthodes, l'unification des types, la construction en série, la 
standardisation ». Les travaux se concluent par la formulation de vœux et le vote de résolutions adoptées par le congrès. Le tout est adressé au ministère du Commerce et de l'Industrie pour une ultime discussion au sein du Comité consultatif des arts et manufactures. L'ensemble des conférences est publié sous la forme de rapports dans dix épais volumes, organisés par sections ${ }^{60}$.

La chimie est évidemment l'objet d'interventions, toutes imprégnées du thème de l'organisation scientifique du travail et de la rationalisation économique, dont témoignent par exemple les propositions d'André Kling sur la réorganisation de l'enseignement de la chimie en France en vue de son adaptation aux nécessités industrielles, celles d'Émile Carron sur la taylorisation dans l'industrie chimique, ou celles, plus explicites encore, d'Émile Fleurent de créer un Conseil d'État technique ${ }^{61}$. Toutes donnent lieu à la rédaction de recommandations conséquentes, validées par le congrès et adressées au Comité consultatif des arts et manufactures.

Dans ses remerciements aux organisateurs du congrès et principaux contributeurs, Clémentel affiche sa satisfaction, motive ses troupes:

"Vous avez été des collaborateurs du gouvernement et je puis vous donner l'assurance que, comme je vous l'avais promis à la Sorbonne, vos vœux ne sont pas allés s'entasser dans les cartons verts de mon administration. Ils ont été placés dans des mains que vous connaissez bien, dont l'activité a déjà été maintes fois applaudie ici, de mon ami Guillet. [...] il va falloir que nous mettions en œuvre bientôt le programme national qui tient en un mot : accroissement de la production ${ }^{62}$. »

\section{L'union industrielle de la science et de l'entreprise}

Chaque secteur d'activité scientifique, économique et industrielle génère ainsi son lot de collaborations avec le ministère, de productions communes d'expertises et de recommandations pour l'avenir. La chimie et son industrie n'échappent nullement à l'exercice auquel vont s'attacher, parmi d'autres, les noms de Haller, Le Chatelier, Eugène Grandmougin, Paul Kestner, Jean-Jacques- Théophile Schloesing, Auguste Béhal ou Fleurent. Tous sont mobilisés d'une façon ou d'une autre dans le but de conduire les études utiles à l'édification du vaste dessein indiqué par Clémentel.

Au titre des réalisations marquantes, il faut ici compter la création de la Société de chimie industrielle 63. Cela avait d'ailleurs été un projet plusieurs fois reporté avant-guerre, mais que le Comité consultatif des arts et manufactures réactive dans ce contexte de réalisation pratique des alliances de la science et de l'industrie. La perspective avait été discutée en son sein, notamment le 4 avril 1917 à partir d'une note de quatre pages exposant les motifs de sa fondation. On y retrouve l'argument d'une nécessaire rénovation économique de la chimie industrielle passant par l'intégration de la science dans les méthodes de fabrication, ou plus généralement par l'établissement d'une liaison intime et permanente entre la science et l'industrie 64.Quelques jours plus tard, le 23 avril 1917, les statuts de l'association sont déposés, avec pour vocation explicite : 1/ de contribuer à l'expansion de l'industrie chimique dans tous ses domaines ; $2 /$ de grouper tous les chefs d'industrie, professeurs, ingénieurs, chimistes, etc. qui y sont intéressés ; 3 / de contribuer au progrès de la chimie industrielle, tant au point de vue économique qu'au point de vue scientifique.

Paul Kestner (1864-1936) en est le premier président. Archétype du chimiste devenu entrepreneur industriel, il incarne l'alliance de la science et de l'industrie. Sa formation est acquise essentiellement auprès de l'École de chimie de Mulhouse. II exerce par la suite dans les laboratoires des usines Kuhlmann, avant d'en assurer la direction. II se fait ensuite luimême entrepreneur industriel et auteur de nombreux brevets. 
Le 16 mars 1918, Kestner inaugure la première séance solennelle sous la présidence de Clémentel. Haller et Le Chatelier sont à ses côtés à titre de présidents d'honneur, rappelant de cette façon que la fondation de cette association s'inscrit résolument dans les plans d'action du Comité consultatif des arts et manufactures et de l'alliance des chimistes. Le ton des premiers instants est clairement orienté par les directives du ministère du Commerce et de I'Industrie, celles qui incitent à la rationalisation de l'économie par la mise au travail collaboratif de la chimie académique et de l'industrie, dont les ingénieurs chimistes sont les agents naturels de mise en œuvre dans les usines et les ateliers ${ }^{65}$. Le $1^{\text {er }}$ juin 1918 , paraît le premier numéro de sa revue mensuelle : Chimie \& Industrie. Elle est dirigée par Camille Matignon, professeur au Collège de France depuis 1908 en remplacement de Le Chatelier. Son article inaugural, signé par Haller, reprend la complainte des chimistes français suite aux expositions de 1893 à Chicago et de 1900 à Paris, martelant l'évidence que « le développement progressif de l'Industrie suit parallèlement celui de la Science elle-même, et les nations où la production intellectuelle est la plus intense, la mieux utilisée, sont celles qui finissent par avoir la suprématie au point de vue industriel 66 ». Kestner explicite sur un ton martial le programme de son journal, que des titres de paragraphes résument en ces termes, dévoilant la teneur du projet volontaire de mobilisation de tout un secteur au service de l'avenir en tous points conformes aux vues du Comité consultatif des arts et manufactures:

« La situation - Ce qui nous attend - Organisons-nous - Des vues d'ensemble - La spécialisation des groupements - Toute la France debout - La marche en commun - De la volonté - De la méthode dans l'effort - Une large documentation - Un office nécessaire - Des missions à l'étranger - L'action régionale - L'utilisation de l'ingénieur chimiste - Le concours des jeunes ${ }^{67}$. »

Un décret du 23 juin attribue la même année à la Société de chimie industrielle le statut d'établissement d'utilité publique. La Société compte alors plus de 2000 membres (elle atteint près de 5000 adhérents en 1925). La composition du conseil d'administration témoigne de sa vocation de franche association industrielle. La plupart de ses membres sont, sinon des administrateurs ou des entrepreneurs, au moins des dirigeants ou directeurs d'exploitation. On retrouve ainsi parmi ses membres les industriels engagés dans les opérations de création ou de restructuration des compagnies chimiques sous l'égide ou l'impulsion du ministère du Commerce et de l'Industrie, ou encore la plupart des entreprises constituant le Syndicat général des produits chimiques ${ }^{68}$.

\section{Conclusion : Un rapport pour organiser la production française}

Les chimistes mobilisés par le ministère dans le giron de cette coalition entre Haller et Le Chatelier, tour à tour, prennent le contrôle du Comité consultatif des arts et manufactures, se font les instigateurs et principaux rapporteurs du congrès du Génie civil, fondent la Société de chimie industrielle. L'alliance de la chimie, de l'industrie et de la rationalisation se concrétise en 1919 par une ultime contribution : la rédaction d'un rapport général commandé par Clémentel. Sous la direction de Guillet, ces chimistes partisans de la rationalisation rédigent les principales et la plupart des recommandations, s'appuyant sur la multitude des études produites sous la férule du ministre.

L'entreprise se traduit en mars par la livraison au président du Conseil, Georges Clemenceau, d'un rapport général sur l'industrie française, regroupant dans un même élan analyses, synthèses, données et recommandations techniques pour l'avenir, devant se traduire par le vote de mesures législatives immédiates et la création d'un comité national d'experts, d'ingénieurs et de techniciens en charge d'éclairer le jugement des responsables politiques ${ }^{69}$.

La chimie et son industrie en tant que telles font évidemment l'objet de recommandations spécifiques, mais elles forment surtout la trame comme le modèle de déploiement des principes de la rationalisation en vue d'un vaste programme de réorganisation de l'économie dans l'après-guerre. La chimie (académique, enseignée, appliquée, industrielle) est 
résolument intégrée aux cadres généraux d'une économie rationnelle, en même temps qu'elle prétend guider son organisation et inviter les chimistes à assurer un rôle politique.

Nouvelle étape de la mobilisation, les élites administratives s'étaient donc faites technocratiques début 1917, entraînées au sein du ministère du Commerce et de l'Industrie par des chimistes résolument partisans de la rationalisation. Leur vœu de renforcer les soubassements scientifiques de la société industrielle devient le projet d'une reconstruction économique guidée par les valeurs du productivisme, la mécanisation à outrance et une direction politique assurée par les hauts cadres techniques de la nation. En 1919, l'heure est à la transition d'une économie de guerre vers celle préconisée par les savants et les ingénieurs chimistes. Dans un rapport rédigé à la suite de l'armistice, Clémentel propose au président de la République la création d'un ministère de la Production nationale et d'une instance pérenne d'experts en charge d'indiquer aux parlementaires la conduite à tenir ${ }^{70}$. Une première réunion du Conseil national économique confirme, le $1^{\text {er }}$ mai 1919, la passation des services techniques du ministère du Commerce à celui de la Reconstitution industrielle confié à Louis Loucheur. L'union visible des chimistes partisans de la rationalisation s'efface dès lors devant la reprise en main par le Parlement de l'économie, mais sans avoir contribué à faire de la rationalisation le mantra d'une alliance entre science, administration et industrie, et dont la chimie et les chimistes furent dans ce contexte particulier le ciment doctrinaire.

\section{NOTES}

1 - Un siècle sépare la publication, en 1819, par le chimiste Jean-Antoine Chaptal, de De l'industrie française et celle, en 1919, du Rapport général sur l'industrie française, à laquelle un autre chimiste, Albin Haller, a largement contribué.

2 - On renvoie ici aux nombreuses études sur la complainte des chimistes durant cette période, de Louis Pasteur à Albin Haller, en passant par Charles Lauth. Voir par exemple commentaires et synthèse proposés par Jun Sakudo, Les Entreprises de la chimie en France de 1860 à 1932, trad. du japonais (Bruxelles : Peter Lang, 2011), 17-118.

3 - Du 4 au 13 septembre, à Washington et New York: Henri Milou, VIII e congrès international de Chimie appliquée (Paris : A. Davy, 1912) ; Huitième congrès international de Chimie appliquée, La Houille blanche, 2 (1913), $46-47$; D. Thorburn Burns et Hendrik Deelsta, The origins and impact of the International Congresses of Applied Chemistry (1894-1912), Microchimica acta, 172/3-4 (2011), 277-283

4 - Robert Richeux, "L'industrie chimique en France : Structure et production », thèse de science économique (Paris, 1958) ; François Crouzet, Essai de construction d'un indiceannuel de la production industrielle française au XIXe siècle, Annales ESC, 25/1 (1970), 56-91.

5 - Henri Hauser, Les Méthodes allemandes d'expansion économique (Paris : Armand Colin, 1915).

6 - John Horne (dir.), State, society, and mobilization in Europe during the First World War (New York : Cambridge University Press, 1997). 7 - François Bouloc, Des temps heureux pour le patronat : La mobilisation industrielle en France, Matériaux pour l'histoire de notre temps, 91/3 (2008), 76-79.

8 - Jean Peyret, L'CEuvre économique du Parlement pendant la guerre : Le rôle de la commission législative du commerce et de l'industrie, ses travaux (1914-1919) (Lyon: Impr. réunies, 1919).

9 - John F. Godfrey, Capitalism at war : Industrial policy and bureaucracy in France (1914-1918) (Leamington Spa : Berg Publishers Ltd., 1987); Gerd Hardach, La mobilisation industrielle en 1914-1918 : Production, planification et idéologie, in Patrick Fridenson(dir.), 1914-1918 : L’autre front (Paris : Les Éditions ouvrières, 1977), « Cahiers du "Mouvement social” », n 2, 81109.

10 - Richard F. Kuisel, Le Capitalisme et l'État en France : Modernisation et dirigisme au XXe siècle (Paris : Gallimard, 1984).

11 - Aimée Moutet, Les Logiques de l'entreprise : La rationalisation dans l'industrie française de l'entre-deux-guerres (Paris : Éd. de l'EHESS, 1997).

12 - Anne Rasmussen, Mobiliser, remobiliser, démobiliser : Les formes d'investissement scientifique en France dans la Grande Guerre, 14-18, 6 (2003), 49-60.

13 - Principal organe technique du ministère du Commerce, le Comité consultatif des arts et manufactures est fondé en 1861.

14 - Christian Topalov (dir.), Laboratoires du nouveau siècle : La nébuleuse réformatrice et ses réseaux en France (1880 1914) (Paris : Éd. de l'EHESS, 1999).

15 - Bruno Marnot, Les Ingénieurs au Parlement sous la Ille République (Paris : Éd. Du CNRS, 2000) ; Michel Pinault, La Science au Parlement : Les débuts d'une politique des recherches scientifiques en France (Paris : Éd. du CNRS, 2000) ; Janet Horne, Le Musée social : Aux origines de l'État-providence (Paris : Belin, 2004) ; Francine Soubiran-Paillet, Parlement, administrateurs et experts (1900-1914) : Le discours de la compétence, Vingtième siècle, 93 (2006), 151-163.

16 - Roy MacLeod et Jeffrey Allan Johnson (dir.), Frontline and factory : Comparative perspectives on the chemical industry at war (1914-1924) (Dordrecht : Springer, 2006), et en particulier Seymour Mauskopf, Revisiting the "chemists 'war"', 1914-1923 : Modern war, munitions, and national systems, 247-255 ; Gerard J. Fitzgerald, Chemical warfare and medical response during World War I, American journal of public health, 98/4 (2008), 611-625.

17 - David Knight et Helge Kragh (dir.), The Making of the chemist : The social history of chemistry in Europe (1789-1914) (Cambridge : Cambridge University Press, 1998). 
18 - AN F12/8053 : Auguste Rateau, « Rapport sur la mécanique », document dactylographié (1918, 53 p.).

19 - Roy MacLeod, L'entente chimique : L'échec de l'avenir à la fin de la guerre (1918- 1922), in David Aubin et Patrice Bret (dir.), Le Sabre et l'éprouvette : L'invention d'une science de guerre (1914-1918) (Paris : Noésis, 2003), 135-152.

20 - Francis Marre, La Chimie meurtrière des Allemands (Paris : Bloud et Gay, 1915).

21 - Olivier Lepick, La Grande guerre chimique (1914-1918) (Paris : Presses universitaires de France, 1998); Annette Becker, La guerre des gaz : Entre tragédie, rumeur, mémoire et oubli, in Christophe Prochasson et Anne Rasmussen, Vrai et faux dans la Grande Guerre (Paris : La Découverte, 2004), 255-276.

22 - Ferdinand George, La Rénovation de l'industrie chimique française : Produits chimiques et pharmaceutiques (Paris : Albin Michel, 1917)

23 - Danielle Fauque, French chemists and the international reorganisation of chemistry after World War I, Ambix, 58/2 (2011), 116-135; Brigitte Schroeder-Gudehus, Les Scientifiques et la paix : La communauté scientifique internationale au cours des années 20 (Montréal : Presses de l'université de Montréal, 1978) ; Anne Rasmussen, Réparer, réconcilier, oublier : Enjeux et mythes de la démobilisation scientifique (1918-1925), Histoire@Politique, 3 (2007), 1-13.

24 - Hervé Joly, La position dominée des chimistes à la direction des grandes firmes chimiques en France (années 1900-1960), in Pierre Lamard et Nicolas Stoskopf, L'Industrie chimique en question (Paris : Alphonse et Jean Picard, 2010), 67-87. 25 - Par exemple, l'incontournable Charles Moureu, La Chimie et la guerre : Science et avenir (Paris : Masson, 1920).

26 - Theo Balderston, Industrial mobilization and war economies, in John Horne (dir.), A companion to the First World War (New York : John Wiley \& Sons, 2010), 217-233 ; Erik Langlinay, Technology, territories and capitalism : World War I and the reshaping of the French chemical industry (1913-1928), in Isabel Malaquias, Ernst Homburg, M. Elvira Callapez (dir.), Chemistry, technology and society : 5th international conference on history of chemistry (6-10 September 2005) (Aveiro : Sociedade portuguesa de química, 2006), 454-466.

27 - Rémy Porte, La Mobilisation industrielle : Premier front de la Grande Guerre ? (Paris :

SOTECA 14-18, 2006) ; Hardach, art. cit. in n. 9.

28 - Eugène et Paul Grandmougin, La Réorganisation de l'industrie chimique en France (Paris : Dunod, 1918) ; Eugène Grandmougin, L'Essor des industries chimiques en France : Ressources et avenir de ces industries : Industries chimiques étrangères (Paris : H. Dunod et E. Pinat, 1917).

29 - Henry Paté, La Loi Dalbiez (Paris : Chapelot, 1915).

30 - Sophie Chauveau, Mobilization and industrial policy : Chemicals and pharmaceuticals in the French war effort, in MacLeod et Johnson (dir.), op. cit. in n. 16, 21-30 ; Thierry Lefebvre, Le Professeur Auguste Béhal et l'Office des produits chimiques et pharmaceutiques (1914-1918), Revue d'histoire de la pharmacie, 81/296 (1993), 71-77.

31 - René-Paul Duchemin, Introduction à l'enquête ouverte par le Syndicat général des produits chimiques sur les modifications à apporter au régime douanier français (Paris : Impr. Le Vauthrin frères, 1916) et Id., L'industrie chimique française (conférence faite à l'École des hautes études sociales le 19 janvier 1925), Bulletin de l'Union des industries chimiques, 2 (1925), 39-56 et 73-86.

32 - Georges Arnaud, Le développement de l'industrie chimique en France, Annales de géographie, 34/191 (1925), 443-445.

33 - Erik Langlinay, Kuhlmann at war (1914-1924), in MacLeod et Johnson (dir.), op. cit. in n. 16, 145-166.

34 - Édouard Bernard, Le Problème de l'azote en France: Agriculture, industrie, défense nationale (Paris : Jean-Baptiste Baillière \& Fils, 1933) ; George Léon, L'Office national industriel de l'azote et le problème de l'azote en France (Paris : Libr. du Recueil Sirey, 1930).

35 - Pierre Renouvin, Les Formes de gouvernement de guerre (Paris : Presses universitaires de France, 1925).

36 - Clotilde Druelle, « Un laboratoire réformateur : Le Département du commerce en France et aux États-Unis de la Grande Guerre aux années vingt », thèse d'histoire (IEP de Paris, 2004) ; Id., De la pensée à l'action économique : Étienne Clémentel (1864- 1936), un ministre visionnaire, Histoire@Politique : Politique, culture, société, 16 (2012), 1-14 (www.histoire-politique.fr). 37 - Stéphane Rials, Administration et organisation (1910-1930) : De l'organisation de la bataille à la bataille de l'organisation dans l'administration française (Paris : Beauchesne, 1977) ; Albert Lanza, Les Projets de réforme administrative en France de 1919 à nos jours (Paris : Presses universitaires de France, 1968).

38 - Fabienne Bock, Un parlementarisme de guerre (1914-1919) (Paris : Belin, 2002) et Id., L'exubérance de l'État en France de 1914 à 1918, Vingtième siècle, 3 (1984), 41- 52 ; Adrian Rossiter, Experiments with corporatist politics in republican France (1916-1939) (Oxford : Oxford University Press, 1987).

39 - Georges-Henri Soutou, L'Or et le sang : Les buts de guerre économiques de la première guerre mondiale (Paris : Fayard, 1989).

40 - Guy Rousseau, Entre idéalisme et réalisme : Une vie politique, Étienne Clémentel (1864-1936) (Clermont-Ferrand : Archives départementales du Puy-de-Dôme, 1998). 41 - Stephen Douglas Carls, Louis Loucheur : Ingénieur, homme d'État, modernisateur de la France (1872-1931) (Villeneuve-d'Ascq : Presses universitaires du Septentrion, 2000); Martin Fine, Albert Thomas : A reformer's vision of modernization (1914- 1932), Journal of contemporary history, 12/3 (1977), 545-564 ; Alain Hennebicque, Albert Thomas et le régime des usines de guerre (1915-1917), in Fridenson (dir.), op. cit. in n. 9, 111-144 ; Bertus Willem Schaper, Albert Thomas : Trente ans de réformisme social (Paris : Presses universitaires de France, 1960).

42 - Pierre Renouvin, La Crise européenne et la Grande Guerre (1904-1914) (Paris: Presses universitaires de France, 1969) ; Raymond Poidevin, Les Relations économiques et financières entre la France et l'Allemagne de 1898 à 1914 (Paris : Armand Colin, 1969) ; Gerald Feldman, Army, industry and labour in Germany (1914-1918) (Londres : Berg Publishers, 1992) ; Hauser, op. cit. in n. 5 et Id., L'Allemagne économique : L'industrie allemande considérée comme facteur de guerre, Bulletin de la Société d'encouragement pour l'industrie nationale, 114 (1915), 425-439.

43 - Claude Digeon, La Crise allemande de la pensée française (Paris : Presses universitaires de France, 1959) ; Albert Sauveur, L'Allemagne et la guerre européenne (Paris : Bloud et Gay, 1916) ; Victor Cambon, L'Allemagne au travail (Paris : P. Roger et $\mathrm{Cie}_{\mathrm{ie}}$ 1917).

44 - Aimée Moutet, La rationalisation : Une réponse aux problèmes de la seconde industrialisation (1880-1939), Bulletin de la SHMC, supplément à la Revue d'histoire moderne et contemporaine, 45 (1998), 1-11.

45 - Charles Maier, Between Taylorism and technocracy : European ideologies and the vision of industrial productivity, Journal of contemporary history, 5/2 (1970), 27-61.

46 - Clothilde Druelle-Korn, « Le ministère du Commerce et de l'Industrie pendant la première guerre mondiale : Étienne Clémentel ou la volonté de moderniser la France ", DEA (IEP de Paris, 1993); Marc Trachtenberg, The Clémentel plan in historical perspective, Proceedings of the annual meeting of the Western Society for French History, 7 (1979), 142-148 et Id., « A new 
economic order » : Étienne Clémentel and French economic diplomacy during the First World War, French historical studies, 10/2 (1977), 315-341.

47 - AN F12/7657 : "Schéma d'organisation des services de guerre du ministère du Commerce » (16 novembre 1917).

48 - Sur le devenir d'une politique de la recherche en lien avec le conflit, voir notamment : Anne-Laure Anizan, Paul Painlevé : Science et politique de la Belle Époque aux années trente (Rennes : Presses universitaires de Rennes, 2012), 123-178 ; Gabriel Galvez-Behar, Le savant, l'inventeur et le politique : Le rôle du sous-secrétariat d'État aux Inventions durant la première guerre mondiale, Vingtième siècle, 85 (2005), 103-117 ; Christine Moissinac et Yves Roussel, Jules-Louis Breton (1878-1940): Un savant parlementaire (Rennes : Presses universitaires de Rennes, 2010), 109-148.

49 - AN F12/7657 : arrêté du 28 septembre 1918.

50 - Voir Erik Langlinay, Albin Haller, chimie et politique (1918-1925), Revue d'histoire des sciences, 69/1 (2016), 117-136 ; Michel Letté, Henry Le Chatelier (1850-1936) ou la science appliquée à l'industrie (Rennes : Presses universitaires de Rennes, 2004).

51 - Léon Guillet est mobilisé par l'Administration au titre des agents fonctionnaires de ce ministère. II ne se verra reconnaître le titre de directeur des services techniques que bien plus tard, par décret du 28 septembre 1918. Sur Guillet : Nicole Chezeau, Léon Guillet, in André Grelon et Claudine Fontanon (dir.) Dictionnaire des professeurs du Conservatoire national des arts et métiers (Paris : INRP et Cnam, 1994).

52 - AN F12/7660 : dossier « Conseil technique » - retranscription de la 10e séance

(25 février 1918).

53 - On trouve sous une forme claire et synthétique la liste des membres et rapporteurs, leurs fonctions, attributions et distribution au sein du Comité consultatif des arts et manufactures dans les premières pages du rapport général : Ministère du Commerce et de l'Industrie, Rapport général sur l'industrie française : Sa situation, son avenir, vol. I (Paris : Imprimerie nationale, 1919), XXV-XXXI. Parmi euX, on retrouve Albin Haller, Henry Le Chatelier, Arsène d'Arsonval, Léon Guillet, Louis de Launay, Auguste Rateau, Léon Lindet, Charles Lauth, Eugène Tisserand, Théophile Schloesing, Émile Fleurent, André Hillairet, Georges Charpy, Henri Hitier, Auguste Béhal, Arthur David- Mennet, Georges Hersent, Arthur Fontaine...

54 - On trouvera, principalement dans les archives du ministère du Commerce et de l'Industrie (AN F12/7657 à 8053), tous les documents de préparation et de réalisation de ses missions. Ici, F12/7657, dossier « Organisation des services constitués au ministère du Commerce et de l'Industrie pendant la durée de la guerre ».

55 - Respectivement : métallurgie (8); textile (11); chimie (52); autres (16).

56 - AN F12/7995 : «Projet de conclusion du discours d'ouverture des travaux économiques du Comité consultatif des arts et manufactures » ; " Projet de programme de travaux destinés à faire suite au discours par M. le ministre à l'ouverture des travaux du Comité consultatif des arts et manufactures » (19 mai 1917).

57 - AN F12/7663 : "Voeux émis par le Comité consultatif des arts et manufactures dans sa séance du 27 mars 1918 sur les articles 419 et 420 du Code pénal - projet de loi sur une modification à apporter à l'article 419 du Code pénal ».

58 - « Congrès général du Génie civil national et interallié : Assises générales de l'industrie pour l'étude et la mise en œuvre des programmes d'après-guerre », Société des ingénieurs civils, Paris, 1917 ; Émile Barbet et Paul Bodin, Premier congrès général du Génie civil national et interallié, La Houille blanche, 4 (1917), 36-39.

59 - AN F12/7994 : Bulletin économique français, 225 (6 mars 1918).

60 - Travaux préparatoires du congrès général du Génie civil, session nationale (mars 1918) (Paris : Hôtel de la Société des ingénieurs civils, 1918), 10 vol. (Rapports présentés à la section [...]) : I. Travaux publics et constructions civiles ; II. Industries des transports ; III. Mécanique, moteurs, machines-outils ; IV. Mines et métallurgie ; V. Physique et chimie industrielles ; VI. Électricité ; VII. Génie rural et industries agricoles ; VIII. Organisation rationnelle du travail industriel ; IX. Hygiène et prévoyance sociale ; $X$. Législation industrielle.

61 - Rapport de M. Kling sur les modifications profondes à faire subir à l'organisation de l'enseignement chimique en France en vue de son adaptation aux nécessités industrielles, in op. cit. in n. 60, section V, 7-13; Rapport de M. Carron sur la taylorisation dans l'industrie chimique, ibid., 17-27 ; Rapport de M. É. Fleurent professeur au Conservatoire national des arts et métiers sur la création d'un Conseil d'État technique, ibid., 154-159.

62 - AN F12/7657 : dossier "Conseil économique », document dactylographié, s. d.

63 - Ulrike Fell, Quelle liaison entre la science et l'industrie ? La Société de chimie industrielle dans l'entre-deux-guerres (19171939), in Ulrike Fell (dir.), Chimie et industrie en Europe : L'apport des sociétés savantes industrielles du XIX e siècle à nos jours (Paris : Éd. des Archives contemporaines, 2001), 69-95.

64 - AN F12/7995: dossier « Société de chimie industrielle ».

65 - Paul Kestner, Inauguration des travaux de la Société de chimie industrielle, Revue scientifique, 56 (1918), 289-304 et Séance d'inauguration des travaux de la Société de chimie industrielle, Chimie \& Industrie, 1 (1918), 32-37 ; Jean Voisin, Le rôle de l'ingénieur chimiste, Chimie \& Industrie, 1 (1918), 566-567.

66 - Albin Haller, La Société de chimie industrielle, Chimie \& Industrie, 1 (1918), 3 (texte daté de décembre 1917).

67 - Paul Kestner, Son programme, Chimie \& Industrie, 1 (1918), 7-9.

68 - Voir la composition complète du bureau de la Société de chimie industrielle, in Son organisation, Chimie \& Industrie, 1 (1918), 10-14.

69 - Michel Letté, Le rapport d'Étienne Clémentel (1919) : L'avènement administratif des technocrates et de la rationalisation, Documents pour l'histoire des techniques, 20 (2011), 167-195.

70 - AN F12/7657, Note pour Monsieur le ministre : « Projet de réorganisation des services de l'administration centrale du ministère du Commerce et de l'Industrie » (novembre 1918). 